\title{
Image Reconstruction by Convolution with Symmetrical Piecewise $n$ th-Order Polynomial Kernels
}

\author{
Erik H. W. Meijering, Karel J. Zuiderveld, Max A. Viergever
}

IEEE Transactions on Image Processing, vol. 8, no. 2, February 1999, pp. 192-201.

\begin{abstract}
The reconstruction of images is an important problem in many applications. From sampling theory it is well known that the sinc function is the ideal interpolation kernel which, however, cannot be used in practice. In order to be able to obtain acceptable reconstructions, both in terms of computational speed and mathematical precision, it is required to design a kernel that is of finite extent and resembles the sinc function as much as possible. In this paper, the applicability of a particular class of sinc-approximating symmetrical piecewise $n$ th-order polynomial kernels is investigated in satisfying these requirements. After the presentation of the general concept, kernels of first, third, fifth and seventh order are derived. An objective, quantitative evaluation of the reconstruction capabilities of these kernels is obtained by analyzing the spatial and spectral behavior using different measures and by using them to translate, rotate and magnify a number of real-life test images. From the experiments it is concluded that while the improvement of cubic convolution over linear interpolation is significant, the use of higher-order polynomials yields only marginal improvement.
\end{abstract}

Keywords - Interpolation, image reconstruction, image resampling, piecewise polynomial kernels, cubic convolution, quintic convolution, septic convolution.

\section{Introduction}

The reconstruction ${ }^{1}$ of images, which are in general $N$-dimensional signals, is an important problem in many applications. Operations such as magnification, subpixel translation, rotation, deformation or warping of images cannot be carried out without reconstructing the image under consideration. Many interpolation schemes have been devised for that purpose. These include the very simple nearest-neighbor and linear interpolators as well as the more computational expensive cubic-convolution [11] and windowed-sinc $[1,15]$ interpolation schemes.

In a particular application, the accuracy with which the original signal needs to be reconstructed from the samples determines which alternative to use. Linear interpolation is computationally very cheap and is satisfactory in many situations. Nowadays, linear interpolation is available as a standard operation in special graphics hardware, making it an attractive option. It has to be expected that as hardware becomes faster, higher-order

\footnotetext{
${ }^{1}$ In this paper, the term "reconstruction" is used to indicate the process of retrieving an original continuous image from its samples. The term "interpolation" is used to indicate the more general process of "filling in" between the samples. That is, any type of interpolation can be used to calculate values at intermediate points. However, in order to reconstruct the values at the intermediate points, specific constraints need to be imposed on the interpolation scheme.
} 
interpolation schemes will become feasible as well. It has been demonstrated $[9,13,15]$ that when further mathematical processing of the data is required, cubic convolution, although more computationally demanding, is a relatively very accurate scheme. The improvement with respect to linear interpolation stimulated us to investigate interpolation kernels consisting of higher-order polynomials than just first or third order.

The purpose of this paper is twofold. First, we will prove that the concept of the two most popular interpolation schemes - linear interpolation and cubic convolutioncan be generalized to a class of what we call symmetrical piecewise nth-order polynomial interpolation kernels. By utilizing this concept, higher-order interpolation kernels will be derived. Secondly, it will be shown that while the improvement of cubic convolution over linear interpolation is substantial, the improvements of higher-order schemes with respect to cubic convolution are only marginal.

The paper is organized as follows. First, in Section II, the concept of $N$-dimensional image reconstruction is presented. It will be shown that the operation can always be carried out by $N$ successive one-dimensional interpolations (cascaded convolution). Next, in Section III, the concept of one-dimensional signal reconstruction by convolution with symmetrical piecewise $n$ th-order polynomial kernels is presented. In Section IV, examples of interpolating kernels consisting of polynomials up to seventh order are given. A quantitative comparison of the reconstruction capabilities of these kernels is presented in Section V. Finally, concluding remarks are made in Section VI.

\section{Reconstruction of $N$-Dimensional Images}

A real-valued continuous image $I(\mathbf{x})$ can be constructed from a real-valued $N$-dimensional discrete image $I_{s}(\mathbf{p})$ by means of interpolation according to:

$$
I(\mathbf{x})=I_{s}(\mathbf{p}) * h(\mathbf{x}),
$$

where $*$ denotes (discrete) convolution, $\mathbf{x}=\left(x_{1}, \ldots, x_{N}\right) \in \mathbb{R}^{N}$ are points in $N$-dimensional continuous space, $\mathbf{p}=\left(p_{1}, \ldots, p_{N}\right) \in \mathbb{Z}^{N}$ are points in $N$-dimensional discrete space (i.e., they are assumed to lie on a regular grid with unit distance between grid points), $I_{s}(\mathbf{p})$ are the image values at those points (the samples), and $h: \mathbb{R}^{N} \rightarrow \mathbb{R}$ is the convolution kernel, interpolating the samples, that is,

$$
I\left(x_{1}, \ldots, x_{N}\right)=\sum_{p_{N}} \cdots \sum_{p_{1}} I_{s}\left(p_{1}, \ldots, p_{N}\right) \cdot h\left(x_{1}-p_{1}, \ldots, x_{N}-p_{N}\right) .
$$

In the Fourier domain this becomes

$$
\widehat{I}(\mathbf{f})=\widehat{I}_{s}(\mathbf{f}) \widehat{H}(\mathbf{f}),
$$

where $\widehat{I}: \mathbb{R}^{N} \rightarrow \mathbb{C}$ is the Fourier transform of $I(\mathbf{x}), \mathbf{f}=\left(f_{1}, \ldots, f_{N}\right) \in \mathbb{R}^{N}$ denotes $N$ dimensional frequency, $\widehat{I}_{s}: \mathbb{R}^{N} \rightarrow \mathbb{C}$ the Fourier transform of the discrete image $I_{s}(\mathbf{x})$ (the sample data), and $\widehat{H}: \mathbb{R}^{N} \rightarrow \mathbb{C}$ the Fourier transform of the convolution kernel.

The question arises whether it is possible to let the interpolated image $I(\mathbf{x})$ become exactly equal to the original continuous version of the sampled image $I_{s}(\mathbf{p})$, prior to sampling. In other words: can the original image be reconstructed from its samples? For one-dimensional signals, the answer is provided by the following well-known theorem.

Theorem 1 Let $I: \mathbb{R} \rightarrow \mathbb{R}$ be a real-valued function that does not contain any frequencies higher than $F_{m}>0$, that is, the Fourier spectrum $\widehat{I}: \mathbb{R} \rightarrow \mathbb{C}$ of that function satisfies 
$\widehat{I}(f)=0,|f|>F_{m}$. Then $I(x), x \in \mathbb{R}$, is completely determined by the samples $I_{s}(p), p \in$ $\mathbb{Z}$ and $I_{s}(p)=I(p)$, if and only if the sampling frequency $F_{s}$ satisfies $F_{s}>2 F_{m}$.

The essence of this theorem has been known to mathematicians in the field of interpolatory function theory since at least 1915 [14] and has later been applied to the fields of telecommunication and information theory by Nyquist [6] and Shannon [12]. An excellent tutorial review on sampling theory has been presented by Jerri [3].

The theorem can easily be extended to $N$-dimensions, in which case $I(\mathbf{x}), \mathbf{x} \in \mathbb{R}^{N}$ can be completely reconstructed from the samples $I_{s}(\mathbf{p}), \mathbf{p} \in \mathbb{Z}^{N}, I_{s}(\mathbf{p})=I(\mathbf{p})$, if and only if the sampling frequencies satisfy $F_{s_{i}}>2 F_{m_{i}}, \forall i=1,2, \ldots, N$, where $F_{m_{i}}$ is the highest frequency in the $i$ th dimension, i.e., $\widehat{I}(\mathbf{f})=0,\left|f_{i}\right|>F_{m_{i}}, \forall i=1,2, \ldots, N$. To this end, the filter $\widehat{H}(\mathbf{f})$ in (3) must be an $N$-dimensional box filter, defined by

$$
\widehat{H}(\mathbf{f})= \begin{cases}1, & \text { if }\left|f_{i}\right| \leqslant \frac{1}{2} F_{s_{i}}, \forall i=1,2, \ldots, N, \\ 0, & \text { if otherwise. }\end{cases}
$$

For a regular grid with unit distance between grid points, the sampling frequencies $F_{s_{i}}$ are equal to 1. Equation (4) can also be written as

$$
\widehat{H}(\mathbf{f})=\prod_{i=1}^{N} \widehat{H}_{B}\left(f_{i}\right),
$$

where $\widehat{H}_{B}: \mathbb{R} \rightarrow \mathbb{R}$ is now a one-dimensional box filter. Thus $\widehat{I}(\mathbf{f})$ becomes

$$
\widehat{I}(\mathbf{f})=\widehat{I}_{s}(\mathbf{f}) \widehat{H}_{B}\left(f_{1}\right) \widehat{H}_{B}\left(f_{2}\right) \cdots \widehat{H}_{B}\left(f_{N}\right) .
$$

The inverse Fourier transform yields

$$
I(\mathbf{x})=\left(\ldots\left(\left(I_{s}(\mathbf{p}) * h_{B}\left(x_{1}\right)\right) * h_{B}\left(x_{2}\right)\right) * \ldots\right) * h_{B}\left(x_{N}\right),
$$

from which it is concluded that $N$-dimensional reconstruction can always be carried out by $N$ successive one-dimensional interpolations. Therefore, in the sequel only the merits of one-dimensional kernels $h(x)$ are investigated.

\section{Symmetrical Piecewise $n$ th-Order Polynomial Kernels}

As shown in the previous section, exact reconstruction of an $N$-dimensional image is accomplished by multiplying the Fourier spectrum $\widehat{I}_{s}(\mathbf{f})$ of the sampled signal $I_{s}(\mathbf{p})$ with an $N$-dimensional box filter or, equivalently, with $N$ successive one-dimensional box filters. By defining the inverse Fourier transform of a spectrum $\widehat{H}(f)$ as $\int_{-\infty}^{\infty} \widehat{H}(f) e^{\mathrm{i} 2 \pi f x} d f$, it can easily be shown that the kernel of the box filter is the sinc function, defined by

$$
h_{B}(x)=\operatorname{sinc}(x) \triangleq \frac{\sin (\pi x)}{\pi x} .
$$

This kernel has infinite extent and cannot be used in practice, thereby ruling out the possibility of exact reconstruction. In order to obtain an acceptable reconstruction (in terms of both computational cost and mathematical precision), it is required to design a kernel of finite extent that resembles the sinc function as much as possible. The purpose of this paper is to investigate to what extent piecewise $n$ th-order polynomial kernels satisfy these requirements. First, the precise definition of these kernels is given. 
Definition 1 Given a regular sampling grid with unit distance between the sample points, the symmetrical piecewise nth-order polynomial interpolation kernel $h(x)$ is defined as

$$
h(x)= \begin{cases}a_{n i}|x|^{n}+\ldots+a_{1 i}|x|+a_{0 i}, & \text { if } i \leqslant|x|<i+1, \\ 0, & \text { if } m \leqslant|x|,\end{cases}
$$

where $i=0,1, \ldots, m-1$, the parameter $m \in \mathbb{N} \backslash\{0\}$ determines the extent of the kernel, and $n$ and $m$ are related by $n=2 m-1 .^{2}$

The $(n+1) m$ coefficients $a_{j i}$ are to be determined by imposing constraints on $h(x)$. Since the samples $I_{s}(\mathbf{p})$ are the exact values of the original image $I(\mathbf{x})$ at the positions $\mathbf{p}$ on the sampling grid, the value of the interpolant at those positions must also be equal to the sample values. Additional constraints are derived by requiring $h(x)$, which consists of piecewise polynomials, to be continuous and, if possible, have continuous derivatives at the transition points $\mathbf{p}$. These requirements can be translated into the constraints

(i) $h(0)=1$ and $h(x)=0$ for $|x|=1, \ldots, m-1$,

(ii) $h^{(l)}(x)$ must be continuous at $|x|=0,1, \ldots, m$,

where the super-script $(l)$ denotes the $l$ th derivative. The second constraint holds for $l=0,1, \ldots, k$, where $k$ must be sufficiently large so as to yield a sufficient number of equations in order to solve for the unknown coefficients $a_{j i}$.

For the determination of an upper limit for $k$, the following lemma is necessary.

Lemma 1 In generating a set of equations according to the aforementioned constraints, in order to solve for the unknown coefficients of the polynomials constituting an interpolation kernel $h(x)$ as given in Definition 1 , the requirement that $h^{(l)}(x)$ must be continuous at $x=0$ does not yield a non-trivial equation in the case $l$ is even.

Proof From the general definition of symmetrical $n$ th-order polynomial kernels it can easily be derived that $h^{(l)}(0)=(l !) a_{l 0}$. The continuity constraint of $h^{(l)}(x)$ at $x=0$, which is expressed as the requirement $\lim _{x \uparrow 0} h^{(l)}(x)=\lim _{x \downarrow 0} h^{(l)}(x)$, leads to $(l !) a_{l 0}=(l !) a_{l 0}$, which does not contain any information. Note that in the case $l$ is odd, the constraint leads to $(l !) a_{l 0}=-(l !) a_{l 0}$, which results in the requirement $a_{l 0}=0$.

By taking into account the result of this lemma, the maximum value for $k$ is given by the following theorem.

Theorem 2 In generating a set of equations according to the aforementioned constraints, in order to solve for the unknown coefficients $a_{j i}$ of the polynomials constituting an interpolation kernel $h(x)$ as given in Definition 1, the maximum allowable value of $k$ is 0 for $n=1$ and $n-2$ for $n>1$.

Proof According to Definition 1 there are $(n+1) m$ unknown coefficients $a_{j i}$, which require the same amount of independent equations in order to be able to obtain a unique solution. The first constraint will result in $m$ equations. The second constraint will yield $(k+1)(m+1)$ equations, and according to Lemma 1, $\left\lceil\frac{k+1}{2}\right\rceil$ of them will not contain information. Hence, the constraints lead to a total of $m+(k+1)(m+1)-\left\lceil\frac{k+1}{2}\right\rceil$ equations. Substituting $k=n-1$ and using the fact that, because $n$ is odd, $n-\left\lceil\frac{n}{2}\right\rceil=\left\lceil\frac{2}{2}\right\rceil-1$, we have $n(m+1)=(n+1) m+(m-1)$ equations. For $n=1, m$ and $n$ are both equal to 1

\footnotetext{
${ }^{2}$ This choice for $n$ will be motivated at the end of this section.
} 
and therefore the number of equations equals the number of unknowns. For $n>1$ we have $n>m$, which results in an over-constrained problem that cannot be solved uniquely, but only e.g. in a least-squares sense. The next smaller integer value is $k=n-2$, which can be derived to yield $(m+1) n-m=(n+1) m-1$ equations.

Corollary $1 \mathrm{If}$, in generating a set of equations according to the aforementioned constraints in order to solve for the unknown coefficients $a_{j i}$ of the polynomials constituting an interpolation kernel $h(x)$ as given in Definition 1, the maximum value for $k$ (according to Theorem 2) is used, the coefficients $a_{j i}$ can be solved uniquely for $n=1$ and are $a$ function of exactly one free parameter, say $\alpha$, for $n>1$.

Corollary 2 If, in generating a set of equations according to the aforementioned constraints in order to solve for the unknown coefficients $a_{j i}$ of the polynomials constituting an interpolation kernel $h(x)$ as given in Definition 1, the maximum value of $k$ (according to Theorem 2) is used, the resulting interpolant will be an element of $C^{0}$ for $n=1$ and of $C^{n-2}$ for $n>1$.

Now that it has been proven that for $n>1$ the coefficients of the polynomials are always a function of a free parameter $\alpha$, that is to say there is a family of possible kernels for every value of $n>1$, it remains to derive a reasonable value for, or at least bounds on, this parameter. An obvious choice would be to require $h^{(k+1)}(x)$ to be continuous at exactly one of the transition points $\mathbf{p}$, which results in exactly one additional equation, thereby allowing the system of equations to be solved uniquely. However, this is not guaranteed to be the best choice when it comes to accurate (mathematical precise) reconstruction. It will be shown that there always exists exactly one optimal value for the parameter $\alpha$. In order to prove this, the following lemma is necessary.

Lemma 2 The Taylor series expansion of the Fourier transform $\widehat{H}(f)$ of a symmetrical piecewise nth-order polynomial interpolation kernel $h(x)$ as given in Definition 1 , in which the coefficients $a_{j i}$ are functions of exactly one free parameter $\alpha$, has the form

$$
\widehat{H}(f)=\beta_{0}(\alpha)+\beta_{2}(\alpha) f^{2}+\beta_{4}(\alpha) f^{4}+\beta_{6}(\alpha) f^{6}+\ldots
$$

That is, the series consists of even terms only, in which the factors $\beta_{c}(\alpha)$ are linear functions of the parameter $\alpha$. In other words, they have the form

$$
\beta_{c}(\alpha)=\zeta_{c_{1}} \alpha+\zeta_{c_{2}} .
$$

Proof Because $h(x)$ is a real-valued function, the Fourier transform $\widehat{H}(f)$ is conjugate symmetric. Furthermore, because $h(x)$ is an even function, the Fourier transform is also even [5]. These two properties lead to the conclusion that $\widehat{H}(f)$ is a real-valued symmetrical function, from which it is known that the Taylor series contains only even terms:

$$
\widehat{H}(f)=\widehat{H}(0)+\frac{1}{2} \widehat{H}^{(2)}(0) f^{2}+\frac{1}{24} \widehat{H}^{(4)}(0) f^{4}+\ldots
$$

By defining the Fourier transform of $h(x)$ as $\int_{-\infty}^{\infty} h(x) e^{-\mathrm{i} 2 \pi f x} d x$, it can easily be derived that $\widehat{H}(f)$ can be written as the sum

$$
\widehat{H}(f)=\sum_{j=0}^{n} \sum_{i=0}^{m-1} a_{j i} \widehat{H}_{j i}(f),
$$


with

$$
\widehat{H}_{j i}(f)=2 \int_{i}^{i+1} x^{j} \cos (2 \pi f x) d x
$$

where use is made of the symmetry property of $h(x)$, and $\mathrm{i}=\sqrt{-1}$ (not to be confused with the index $i$ ). According to (13), the derivatives in (12) are calculated as

$$
\widehat{H}^{(l)}(0)=\sum_{j=0}^{n} \sum_{i=0}^{m-1} a_{j i} \widehat{H}_{j i}^{(l)}(0),
$$

from which it is concluded that the factors $\beta_{c}(\alpha)$ in the series are linear combinations of the coefficients $a_{j i}$, which are in turn linear combinations of the parameter $\alpha$.

Theorem 3 The value of the free parameter $\alpha$ that yields the most mathematical precise interpolant $h(x)$ can be determined by taking

$$
\beta_{2}(\alpha)=0 .
$$

Proof In order to prevent high-frequency emphasis in the Fourier spectrum $\widehat{H}(f)$ of the interpolation kernel $h(x)$, the value of the parameter $\alpha$ should be chosen so as to force $\widehat{H}(f)$ to be concave downward at $f=0$. This is expressed in the requirement

$$
\left.\frac{d^{2} \widehat{H}(f)}{d f^{2}}\right|_{f=0} \leqslant 0 .
$$

In order to prevent low-frequency suppression in the Fourier spectrum $\widehat{H}(f)$ of the interpolation kernel $h(x)$, the value of the parameter $\alpha$ should be chosen so as to force $\widehat{H}(f)$ to be concave upward at $f=0$. This is expressed in the requirement

$$
\left.\frac{d^{2} \widehat{H}(f)}{d f^{2}}\right|_{f=0} \geqslant 0 .
$$

This leads to the conclusion that the optimal value for the free parameter $\alpha$ is the one that satisfies both requirements (17) and (18), and since

$$
\left.\frac{d^{2} \widehat{H}(f)}{d f^{2}}\right|_{f=0}=2 \beta_{2}(\alpha),
$$

this implies that the optimal value can be found by taking $\beta_{2}(\alpha)=0$.

In Definition 1, the order $n$ of the polynomials was related to the extent $m$ by $n=$ $2 m-1$. Since $m \in \mathbb{N} \backslash\{0\}$, this implies that $n$ will always be odd. It has to be pointed out that $n=2 m-1$ is not the only possible value for $n$. In fact, it can be shown that $n=2 m$ or $n=2 m+1$ will also lead to a unique kernel for all $m \in \mathbb{N} \backslash\{0\}$. In many cases, even higher values for $n$ are possible. However, given the extent $m$, the value $n=2 m-1$ is the lowest possible order resulting in one-parameter polynomials for which a unique solution exists, using the approach described in this section. In order to solve for the unknown coefficients in the case that $n>2 m-1$, higher-order derivatives are required to be continuous ( $k$ must be larger). However, a larger value for $k$ implies that the resulting kernel will be smoother than its lower-order version with the same extent, causing both its spatial and spectral behavior to be worse. 


\section{Kernel Examples}

The theory developed in the previous section will now be used to derive the four lowestorder piecewise polynomial interpolation kernels, which provide for linear, cubic, quintic and septic interpolation.

\section{IV.A Linear Interpolation}

The kernel of the linear interpolator consists of linear polynomials and approximates the ideal sinc function in the interval $[-1,1]$. The kernel is given by

$$
h_{L}(x)= \begin{cases}a_{10}|x|+a_{00}, & \text { if } 0 \leqslant|x|<1 \\ 0, & \text { if } 1 \leqslant|x| .\end{cases}
$$

The two coefficients $a_{j i}$ can be derived by imposing the constraints

(i) $h_{L}(0)=1$,

(ii) $h_{L}(x)$ must be continuous at $|x|=0,1$.

These constraints yield two equations in the two unknown coefficients $a_{j i}$. This system can be solved uniquely, yielding $a_{10}=-1$ and $a_{00}=1$. The resulting interpolant will be an element of $C^{0}$ (that is, it will be continuous).

\section{IV.B Cubic Convolution}

The cubic-convolution kernel consists of third-order polynomials and approximates the ideal sinc function in the interval $[-2,2]$. The kernel is given by

$$
h_{C}(x)= \begin{cases}a_{30}|x|^{3}+a_{20}|x|^{2}+a_{10}|x|+a_{00}, & \text { if } 0 \leqslant|x|<1, \\ a_{31}|x|^{3}+a_{21}|x|^{2}+a_{11}|x|+a_{01}, & \text { if } 1 \leqslant|x|<2, \\ 0, & \text { if } 2 \leqslant|x| .\end{cases}
$$

The eight coefficients $a_{j i}$ can be derived by imposing the constraints

(i) $h_{C}(0)=1$ and $h_{C}(x)=0$ for $|x|=1$,

(ii) $h_{C}^{(l)}(x)$ must be continuous at $|x|=0,1,2$ for $l=0,1$.

These constraints yield seven equations in eight unknown coefficients $a_{j i}$. By allowing $a_{31}=\alpha$ to be a tunable parameter, the system can be solved, yielding the values for the eight coefficients as shown in Table 1.

The Taylor series around $f=0$ of the Fourier spectrum of this kernel is given by

$$
\widehat{H}_{C}(f)=1-\frac{4}{15}(2 \alpha+1)(\pi f)^{2}+\frac{1}{35}(16 \alpha+1)(\pi f)^{4}+\mathcal{O}\left(f^{6}\right) .
$$

By imposing the constraints that the lower frequencies are not allowed to be suppressed and that the higher frequencies are not allowed to be amplified, we find that the best cubic-convolution kernel is the one with a value for $\alpha$ such that $\widehat{H}_{C}(f)$ is neither convex nor concave at $f=0$, that is, the one for which the second-order term in (22) vanishes:

$$
\left.\frac{d^{2} \widehat{H}_{C}(f)}{d f^{2}}\right|_{f=0}=-\frac{8}{15}(2 \alpha+1)=0,
$$

which yields $\alpha=-1 / 2[4,8]$. The resulting interpolant will be an element of $C^{1}$ (that is, it will be continuous and will have a continuous first derivative). 


\begin{tabular}{|r||r|r|r|r|}
\hline$i$ & $a_{3 i}$ & $a_{2 i}$ & $a_{1 i}$ & $a_{0 i}$ \\
\hline \hline 0 & $\alpha+2$ & $-(\alpha+3)$ & 0 & 1 \\
\hline 1 & $\alpha$ & $-5 \alpha$ & $8 \alpha$ & $-4 \alpha$ \\
\hline
\end{tabular}

Table 1. The eight coefficients of the polynomials of the cubic-convolution kernel as a function of the free parameter $\alpha$.

\begin{tabular}{|r||r|r|r|r|r|r|}
\hline$i$ & $a_{5 i}$ & $a_{4 i}$ & $a_{3 i}$ & $a_{2 i}$ & $a_{1 i}$ & $a_{0 i}$ \\
\hline \hline 0 & $10 \alpha-\frac{21}{16}$ & $-18 \alpha+\frac{45}{16}$ & 0 & $8 \alpha-\frac{5}{2}$ & 0 & 1 \\
\hline 1 & $11 \alpha-\frac{5}{16}$ & $-88 \alpha+\frac{45}{16}$ & $270 \alpha-10$ & $-392 \alpha+\frac{35}{2}$ & $265 \alpha-15$ & $-66 \alpha+5$ \\
\hline 2 & $\alpha$ & $-14 \alpha$ & $78 \alpha$ & $-216 \alpha$ & $297 \alpha$ & $-162 \alpha$ \\
\hline
\end{tabular}

Table 2. The 18 coefficients of the polynomials of the quintic-convolution kernel as a function of the free parameter $\alpha$.

\section{IV.C Quintic Convolution}

The quintic-convolution kernel consists of fifth-order polynomials and approximates the ideal sinc function in the interval $[-3,3]$. The kernel is given by

$$
h_{Q}(x)= \begin{cases}a_{50}|x|^{5}+\ldots+a_{10}|x|+a_{00}, & \text { if } 0 \leqslant|x|<1, \\ a_{51}|x|^{5}+\ldots+a_{11}|x|+a_{01}, & \text { if } 1 \leqslant|x|<2, \\ a_{52}|x|^{5}+\ldots+a_{12}|x|+a_{02}, & \text { if } 2 \leqslant|x|<3, \\ 0, & \text { if } 3 \leqslant|x| .\end{cases}
$$

The 18 coefficients $a_{j i}$ can be derived by imposing the constraints

(i) $h_{Q}(0)=1$ and $h_{Q}(x)=0$ for $|x|=1,2$,

(ii) $h_{Q}^{(l)}(x)$ must be continuous at $|x|=0,1,2,3$ for $l=0,1,2,3$.

These constraints yield 17 equations in the 18 unknown coefficients $a_{j i}$. By allowing $a_{52}=\alpha$ to be a tunable parameter, the system can be solved, yielding the values for the 18 coefficients as shown in Table 2.

The Taylor series around $f=0$ of the Fourier spectrum of this kernel is given by

$$
\widehat{H}_{Q}(f)=1+\frac{1}{14}(64 \alpha-3)(\pi f)^{2}-\frac{1}{105}(352 \alpha+1)(\pi f)^{4}+\mathcal{O}\left(f^{6}\right) .
$$

By imposing the constraint that the Fourier spectrum $\widehat{H}_{Q}(f)$ is neither convex nor concave at $f=0$, we find that the best quintic-convolution kernel is the one for which the secondorder term in (25) vanishes:

$$
\left.\frac{d^{2} \widehat{H}_{Q}(f)}{d f^{2}}\right|_{f=0}=\frac{2}{14}(64 \alpha-3)=0,
$$

which yields $\alpha=3 / 64$. The resulting interpolant will be an element of $C^{3}$ (that is, it will be continuous and will have continuous derivatives up to third order). 


\begin{tabular}{|r||r|r|r|r|}
\hline$i$ & $a_{7 i}$ & $a_{6 i}$ & $a_{5 i}$ & $a_{4 i}$ \\
\hline \hline 0 & $245 \alpha+\frac{821}{1734}$ & $-621 \alpha-\frac{1148}{867}$ & 0 & $760 \alpha+\frac{1960}{867}$ \\
\hline 1 & $301 \alpha+\frac{1687}{6936}$ & $-3309 \alpha-\frac{2492}{867}$ & $14952 \alpha+\frac{32683}{2312}$ & $-35640 \alpha-\frac{128695}{3468}$ \\
\hline 2 & $57 \alpha+\frac{35}{6936}$ & $-1083 \alpha-\frac{175}{1734}$ & $8736 \alpha+\frac{1995}{2312}$ & $-38720 \alpha-\frac{4725}{1156}$ \\
\hline 3 & $\alpha$ & $-27 \alpha$ & $312 \alpha$ & $-2000 \alpha$ \\
\hline \hline$i$ & $a_{3 i}$ & $a_{1 i}$ & $a_{0 i}$ \\
\hline \hline 0 & 0 & $-384 \alpha-\frac{1393}{578}$ & 0 & 1 \\
\hline 1 & $47880 \alpha+\frac{127575}{2312}$ & $-36000 \alpha-\frac{13006}{289}$ & $14168 \alpha+\frac{120407}{6936}$ & $-2352 \alpha-\frac{2233}{1156}$ \\
\hline 2 & $101640 \alpha+\frac{1575}{136}$ & $-157632 \alpha-\frac{5670}{289}$ & $133336 \alpha+\frac{42525}{2312}$ & $-47280 \alpha-\frac{8505}{1156}$ \\
\hline 3 & $7680 \alpha$ & $-17664 \alpha$ & $22528 \alpha$ & $-12288 \alpha$ \\
\hline
\end{tabular}

Table 3. The 32 coefficients of the polynomials of the septic-convolution kernel as a function of the free parameter $\alpha$.

\section{IV.D Septic Convolution}

The septic-convolution kernel consists of seventh-order polynomials and approximates the ideal sinc function in the interval $[-4,4]$. The kernel is given by

$$
h_{S}(x)= \begin{cases}a_{70}|x|^{7}+\ldots+a_{10}|x|+a_{00}, & \text { if } 0 \leqslant|x|<1, \\ a_{71}|x|^{7}+\ldots+a_{11}|x|+a_{01}, & \text { if } 1 \leqslant|x|<2, \\ a_{72}|x|^{7}+\ldots+a_{12}|x|+a_{02}, & \text { if } 2 \leqslant|x|<3, \\ a_{73}|x|^{7}+\ldots+a_{13}|x|+a_{03}, & \text { if } 3 \leqslant|x|<4, \\ 0, & \text { if } 4 \leqslant|x| .\end{cases}
$$

The 32 coefficients $a_{j i}$ can be derived by imposing the constraints

(i) $h_{S}(0)=1$ and $h_{S}(x)=0$ for $|x|=1,2,3$,

(ii) $h_{S}^{(l)}(x)$ must be continuous at $|x|=0,1,2,3,4$ for $l=0,1,2,3,4,5$.

These constraints yield 31 equations in the 32 unknown coefficients $a_{j i}$. By allowing $a_{73}=\alpha$ to be a tunable parameter, the system can be solved, yielding the values for the 32 coefficients as shown in Table 3.

The Taylor series around $f=0$ of the Fourier spectrum of this kernel is given by

$$
\widehat{H}_{S}(f)=1-\frac{2}{867}(83232 \alpha+71)(\pi f)^{2}+\frac{8}{8415}(97920 \alpha-53)(\pi f)^{4}+\mathcal{O}\left(f^{6}\right) .
$$

Again, by imposing the constraint that the magnitude of the Fourier spectrum $\widehat{H}_{S}(f)$ is neither convex nor concave at $f=0$, we find that the best septic-convolution kernel is the one for which the second-order term in (28) vanishes:

$$
\left.\frac{d^{2} \widehat{H}_{S}(f)}{d f^{2}}\right|_{f=0}=-\frac{4}{867}(83232 \alpha+71)=0,
$$

which yields $\alpha=-71 / 83232$. The resulting interpolant will be an element of $C^{5}$ (that is, it will be continuous and wil have continuous derivatives up to fifth order). 


\section{Experimental Results}

In this section, the reconstruction capabilities of the kernels derived in the previous section will be quantitatively evaluated and compared. First, both the spatial and spectral behavior of the kernels will be analyzed. Next, the kernels will be applied in a number of experiments on real-life images. Finally, the results will be briefly discussed.

\section{V.A Spatial and Spectral Analyses}

Plots of the four interpolation kernels $h_{L}(x), h_{C}(x), h_{Q}(x)$, and $h_{S}(x)$, as presented in the previous section, are shown in Figure 1, together with the plots of their spectra $\widehat{H}_{L}(f)$, $\widehat{H}_{C}(f), \widehat{H}_{Q}(f)$ and $\widehat{H}_{S}(f)$. From these plots it can be observed that the cubic-convolution kernel resembles the ideal box filter $\widehat{H}_{B}(f)$ substantially better than the linear interpolation kernel. The spectra of the quintic- and septic-convolution kernels appear identical to that of the cubic-convolution scheme. However, their corresponding log-plots in Figure 1 reveal that the high-frequency suppression capabilities of the quintic- and septicconvolution kernels are, respectively, one and two orders of magnitude better than that of the cubic-convolution kernel.

Since the spectra of the kernels are all flat at $f=0$ (because of the constraint proposed in Theorem 3), they can be compared by evaluating the transition rate from stop-band $(|f|>1 / 2)$ to pass-band $(-1 / 2 \leqslant f \leqslant 1 / 2)$. This rate corresponds to the first derivative of the spectrum $\widehat{H}(f)$ at $f=-1 / 2$, and should be as large as possible. The values of this derivative for the presented kernels are shown in Table 4. The ideal kernel (the sinc function) has an infinite transition rate, causing high frequencies to be completely suppressed and low frequencies to be completely conserved. The improvement of cubic convolution over linear interpolation is $43.2 \%$ according to this feature. Quintic and septic convolution are respectively $49.2 \%$ and $56.6 \%$ better than linear interpolation, but only $4.2 \%$ and $9.3 \%$ better than cubic convolution.

In the literature on sampling and reconstruction, an important error measure to study the spectral behavior of a kernel as a function of frequency is given by

$$
E(f)=|1-\widehat{H}(f)|^{2}+\sum_{\eta \in \mathbb{Z} \backslash\{0\}}\left|\widehat{H}\left(f-\eta F_{s}\right)\right|^{2},
$$

where $\widehat{H}(f), f \in \mathbb{R}$ denotes the spectrum of the kernel to be analyzed and $F_{s} \in \mathbb{R}$ is the sampling frequency. This measure was originally proposed by Park \& Schowengerdt [7] in studying the blur caused by sampling and reconstruction (SR-blur) and has since been applied by several others $[2,10]$ to compare their methods to cubic convolution. Note that for the sinc function, $E(f)=0$ for $|f| \leqslant F_{s} / 2$ and $E(f)=2$ for $|f|>F_{s} / 2$. The error functions $E(f)$ for the spectra of the four interpolation kernels are shown in Figure 2, together with that of the sinc function. From this figure it is clear that the cubic-convolution kernel is substantially better than the linear interpolation kernel, but the improvements of the higher-order kernels are only marginal.

We also computed the total square error (distance) of the spectra $\widehat{H}(f)$ with respect to the spectrum $\widehat{H}_{B}(f)$ of the sinc function,

$$
E_{T}=\int_{-\infty}^{\infty}\left|\widehat{H}_{B}(f)-\widehat{H}(f)\right|^{2} d f=\int_{-\frac{1}{2}}^{\frac{1}{2}} E(f) d f .
$$



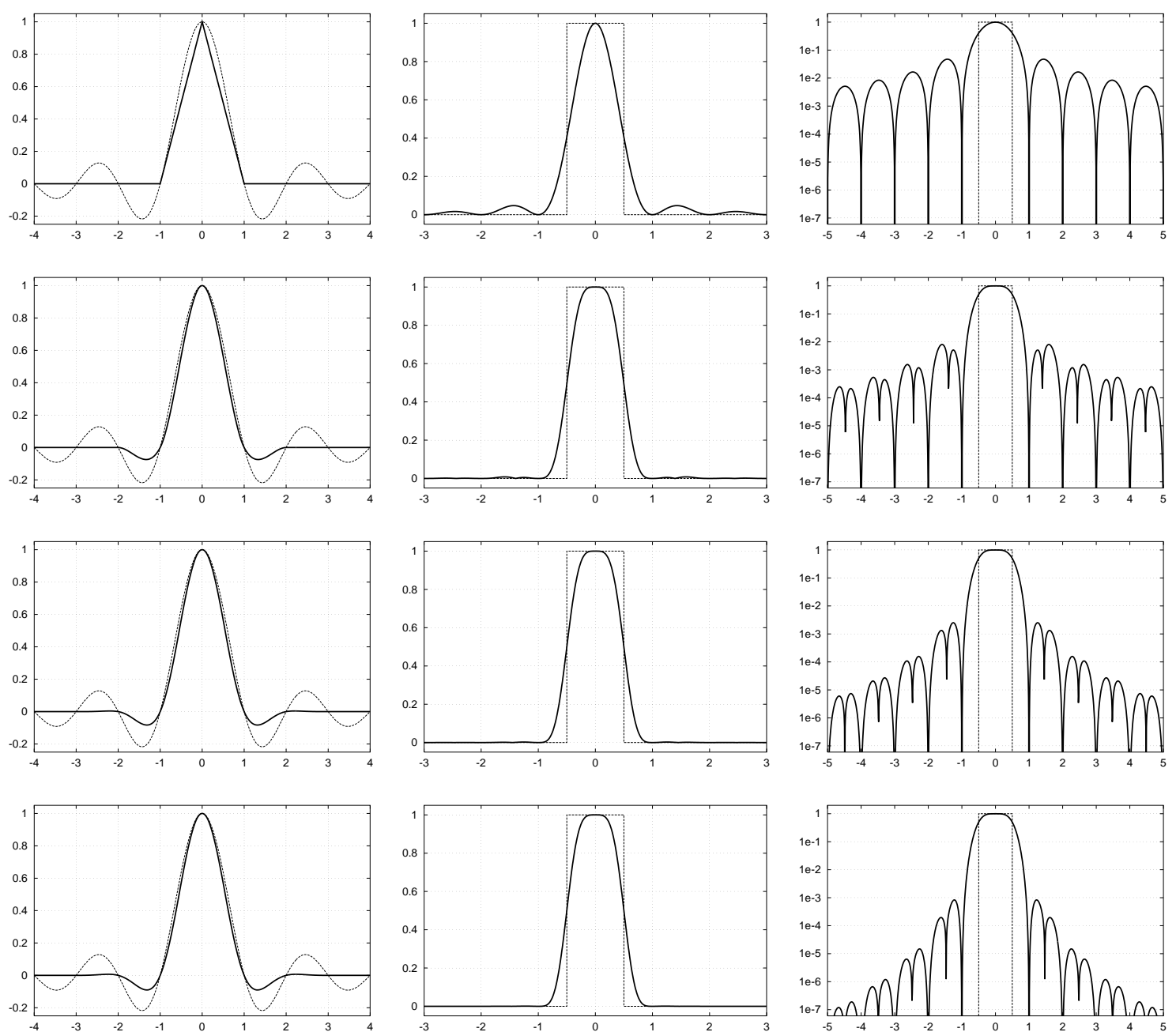

Figure 1. The kernels of the four lowest-order interpolators, compared to the ideal interpolation kernel: the sinc function (dashed line). First column: the linear interpolation kernel $h_{L}(x)$, the cubic-convolution kernel $h_{C}(x)$, the quintic-convolution kernel $h_{Q}(x)$ and the septic-convolution kernel $h_{S}(x)$. Second column: the corresponding Fourier spectra $\widehat{H}_{L}(f)$, $\widehat{H}_{C}(f), \widehat{H}_{Q}(f)$ and $\widehat{H}_{S}(f)$, compared to the spectrum $\widehat{H}_{B}(f)$ of the sinc function (dashed line). Third column: the corresponding log-plots of the spectra.

The results for the four interpolation kernels are listed in Table 5. From these figures it can be concluded that according to the $E_{T}$ error measure, the improvement of cubic convolution over linear interpolation is $33.9 \%$. Quintic and septic convolution are respectively $36.4 \%$ and $39.2 \%$ better than linear interpolation, but only $3.8 \%$ and $8.0 \%$ better than cubic convolution.

\section{V.B Evaluation using Real-Life Images}

In order to obtain a quantitative comparison of the performance of the four interpolation kernels when applied to real-life images, the kernels were used in three different types of operations, each of which requires accurate reconstruction: (i) subpixel translation, 


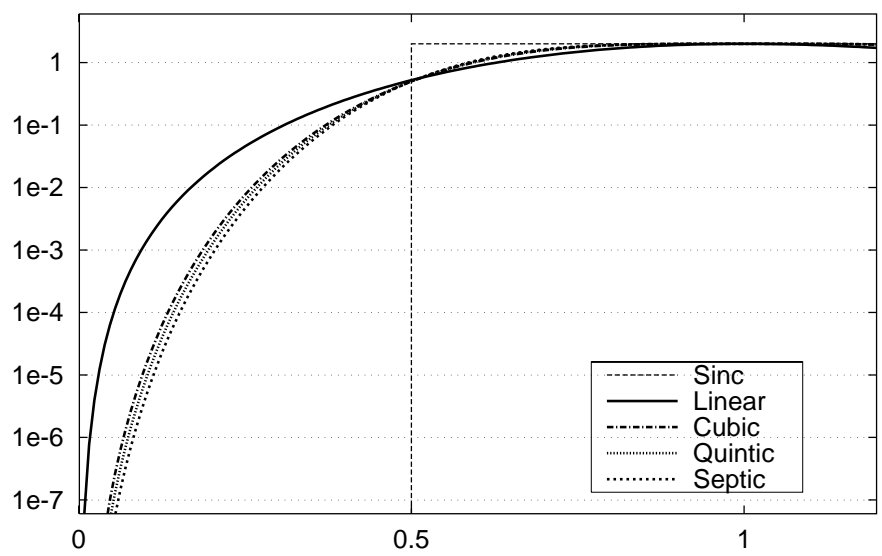

Figure 2. The error function $E(f)$ (30) for the spectra of the four interpolation kernels described in Section IV, compared to that of the sinc function.

\begin{tabular}{|l||r|}
\hline Kernel & $\widehat{H}^{(1)}\left(-\frac{1}{2}\right)$ \\
\hline \hline$h_{L}(x)$ & 1.621 \\
\hline$h_{C}(x)$ & 2.321 \\
\hline$h_{Q}(x)$ & 2.419 \\
\hline$h_{S}(x)$ & 2.538 \\
\hline$h_{B}(x)$ & $\infty$ \\
\hline
\end{tabular}

Table 4. The first derivative of the Fourier transform $\widehat{H}(f)$ of the four interpolation kernels $h_{L}(x), h_{C}(x), h_{Q}(x)$, and $h_{S}(x)$ presented in Section IV, at the transition from stop-band to pass-band $(f=-1 / 2)$, compared to that of the sinc function $h_{B}(x)$.

\begin{tabular}{|l||r|}
\hline Kernel & $E_{T}$ \\
\hline \hline$h_{L}(x)$ & 0.119277 \\
\hline$h_{C}(x)$ & 0.078894 \\
\hline$h_{Q}(x)$ & 0.075913 \\
\hline$h_{S}(x)$ & 0.072559 \\
\hline$h_{B}(x)$ & 0 \\
\hline
\end{tabular}

Table 5. The total square error $E_{T}$ (31) of the spectra of the four interpolation kernels $h_{L}(x), h_{C}(x), h_{Q}(x)$, and $h_{S}(x)$ presented in Section IV, compared to that of the spectrum of the sinc function $h_{B}(x)$.

(ii) rotation, and (iii) magnification. For these experiments, the 16 two-dimensional test images shown in Figure 3 were used. ${ }^{3}$

To carry out a subpixel translation, the following two operations have to be performed: (i) reconstruction of the image, and (ii) sampling of the reconstructed image at a new, translated grid. The first operation requires interpolation for which, in this experiment, the four schemes presented in Section IV were used. The translated images should be

${ }^{3}$ In all three experiments, the images were mirrored around the borders to reduce border artifacts. 

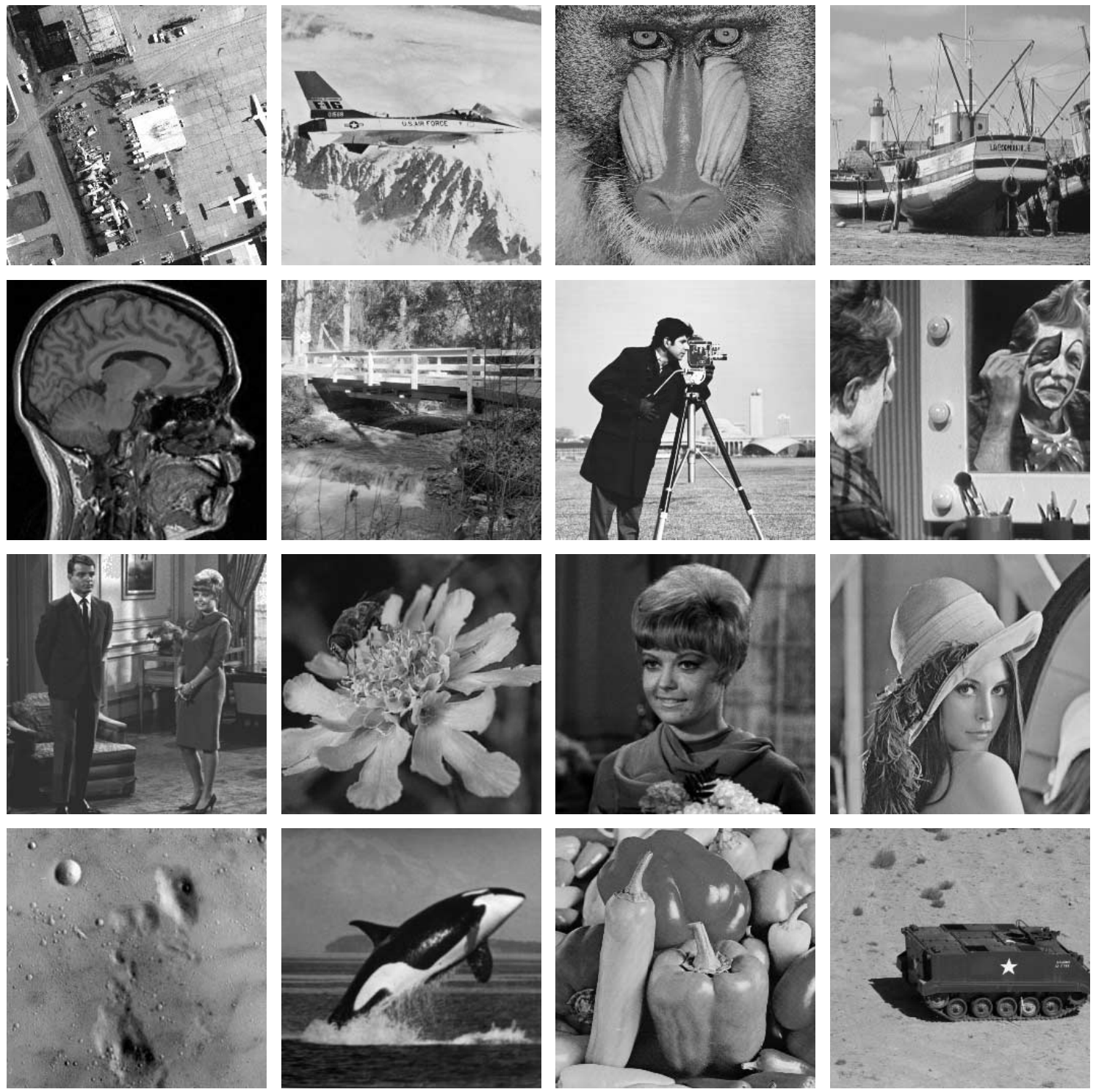

Figure 3. The 16 test images used in the experiments for comparison of the four interpolation schemes described in Section IV. From top-left to bottom-right: Airfield, Airplane, Baboon, Boat, Brain, Bridge, Camera, Clown, Couple, Flower, Girl, Lena, Moon, Orca, Peppers and Tank.

compared to the corresponding ideally translated one (in which the sinc function is used as interpolation kernel). Since such an image cannot be obtained, the images were translated back to their initial position (using the same interpolation kernel as for the forward translation) and the mean square error with respect to the original (non-translated) version was computed. The results of this subpixel translation experiment for the 16 test images and for a displacement of $(0.4,0.7)$ pixel are presented in Table 6. According to these figures, the improvement of cubic convolution over linear interpolation is (on average) $65.1 \%$. Quintic and septic convolution are, respectively, $67.6 \%$ and $69.9 \%$ better than linear interpolation, but only $7.6 \%$ and $14.3 \%$ better than cubic convolution. 


\begin{tabular}{|c|c|c|c|c|c|c|c|c|c|c|c|c|}
\hline \multirow[t]{3}{*}{ Image } & \multicolumn{12}{|c|}{ Experiment } \\
\hline & \multicolumn{4}{|c|}{ Subpixel Translation } & \multicolumn{4}{|c|}{ Rotation } & \multicolumn{4}{|c|}{ Magnification } \\
\hline & $\overline{h_{L}}$ & $h_{C}$ & $\overline{h_{Q}}$ & $h_{S}$ & $\overline{h_{L}}$ & $h_{C}$ & $\overline{h_{Q}}$ & $\overline{h_{S}}$ & $\overline{h_{L}}$ & $h_{C}$ & $\overline{h_{Q}}$ & $\overline{h_{S}}$ \\
\hline \begin{tabular}{|l|l} 
AIRFIELD \\
\end{tabular} & 78.85 & \begin{tabular}{|l|}
29.88 \\
\end{tabular} & 27.78 & 25.97 & $\overline{52.21}$ & 18.13 & $\overline{16.83}$ & 15.79 & 90.79 & $\mid 54.74$ & 52.74 & 50.53 \\
\hline \begin{tabular}{|l} 
AIRPLANE \\
\end{tabular} & 16.07 & 4.09 & 3.73 & 3.39 & 10.29 & 2.34 & 2.12 & 1.93 & 43.09 & 22.81 & 21.69 & 20.45 \\
\hline BABOON & 154.12 & 63.85 & 58.98 & 55.29 & 107.05 & 42.32 & 39.42 & 37.12 & 46.22 & 28.69 & 27.62 & 26.43 \\
\hline BOAT & 28.10 & 9.95 & 9.21 & 8.42 & 17.03 & 4.77 & 4.35 & 3.97 & 42.71 & 25.56 & 24.56 & 23.45 \\
\hline BRAIN & 32.26 & 6.20 & 5.54 & 4.90 & 20.09 & 3.47 & 3.09 & 2.75 & 95.57 & 55.10 & 52.84 & 50.36 \\
\hline BRIDGE & 98.23 & 42.84 & 39.98 & 37.69 & 65.59 & 25.40 & 23.67 & 22.32 & 73.48 & 44.61 & 42.91 & \begin{tabular}{|l|}
41.01 \\
\end{tabular} \\
\hline \begin{tabular}{|l|} 
CAMERA \\
\end{tabular} & 119.99 & 51.27 & 47.98 & 45.01 & 76.01 & 27.30 & 25.33 & 23.74 & 87.97 & 51.36 & 49.35 & \begin{tabular}{|l|}
47.11 \\
\end{tabular} \\
\hline CLOWN & 34.22 & 8.54 & 7.79 & 7.03 & 21.45 & 4.75 & 4.31 & 3.91 & 91.22 & 57.82 & 55.95 & 53.88 \\
\hline COUPle & 41.67 & 14.83 & 13.72 & 12.74 & 26.62 & 8.29 & 7.63 & $\overline{7.09}$ & 56.59 & 36.51 & 35.31 & 33.97 \\
\hline \begin{tabular}{|l} 
FLOWER \\
\end{tabular} & 7.10 & 2.20 & 2.04 & 1.91 & 4.69 & 1.38 & 1.29 & 1.22 & 25.91 & 13.89 & 13.29 & 12.63 \\
\hline GIRL & 18.09 & 6.33 & 5.87 & 5.48 & 12.14 & 3.92 & 3.63 & 3.41 & 44.14 & 31.57 & 30.89 & 30.13 \\
\hline LENA & 14.98 & 5.02 & 4.67 & 4.36 & 9.49 & 2.78 & 2.57 & 2.39 & 31.75 & \begin{tabular}{|l|}
18.78 \\
\end{tabular} & 18.10 & 17.36 \\
\hline MOON & 13.52 & 5.92 & 5.53 & 5.19 & 8.46 & 3.06 & 2.83 & 2.64 & 11.14 & 6.78 & 6.53 & 6.27 \\
\hline ORCA & 5.83 & 1.14 & 1.03 & 0.93 & 3.90 & 0.71 & 0.64 & 0.58 & 29.59 & 14.84 & 14.11 & 13.32 \\
\hline PEPPERS & 17.43 & 8.19 & $\overline{7.74}$ & 7.38 & 12.00 & 5.25 & 4.99 & 4.80 & 30.23 & \begin{tabular}{|l|}
18.17 \\
\end{tabular} & 17.59 & \begin{tabular}{|l|}
16.94 \\
\end{tabular} \\
\hline TANK & 20.76 & 8.81 & 8.19 & $\overline{7.72}$ & 14.33 & 5.62 & 5.25 & 4.96 & 16.50 & 10.35 & 10.02 & 9.65 \\
\hline
\end{tabular}

Table 6. The mean square errors (MSE) made by the four interpolation kernels when using them in subpixel translation, rotation, and magnification operations, applied to the 16 real-life test images shown in Figure 3. The translation was carried out over the vector $(0.4,0.7)$, the rotation was over $15^{\circ}$, and the magnification was done with a factor of 4 .

As a second experiment, the test images were rotated. In order to rotate an image, the two following operations need to be carried out: (i) reconstruction of the image, and (ii) sampling of the reconstructed image at a new, rotated grid. The first operation requires interpolation, for which the four schemes presented in Section IV were used. The rotated images should be compared to the corresponding ideally rotated one. Since such an image cannot be obtained, the images were rotated back to their initial orientation (using the same interpolation kernel as for the forward rotation) and the mean square error with respect to the original version was computed. The results of this experiment for the 16 test images and for a rotation angle of $15^{\circ}$ are presented in Table 6 . According to these figures, the improvement of cubic convolution over linear interpolation is (on average) $68.8 \%$. Quintic and septic convolution are, respectively, $71.2 \%$ and $73.1 \%$ better than linear interpolation, but only $7.8 \%$ and $14.3 \%$ better than cubic convolution.

Finally, the test images were magnified. In order to magnify an image, the following three operations have to be carried out: (i) reconstruction of the image, (ii) sampling of the reconstructed image at a new, more dense grid, and (iii) scaling of the resampled image. The first operation requires interpolation for which, again, the four schemes presented in Section IV were used. The magnified images should be compared to the corresponding ideally magnified one. Since such an image cannot be obtained and since the inverse operation (i.e., subsampling) would yield exactly the original image (by definition of the kernels, recall the constraints in Section III), we had to resort to a different evaluation strategy: the magnification operation was applied to subsampled versions of the original images which, in order to reduce the influence of aliasing, were low-pass filtered prior to subsampling. That is, the low-pass filtered versions of the 16 test images (Figure 3) were taken as the new originals and were then successively subsampled with a factor 4 and magnified with the same factor. The magnified images (now having the same size as the 
original images) were compared to the low-pass filtered versions by computing the mean square error. The results of this experiment are also listed in Table 6. According to these figures, the improvement of cubic convolution over linear interpolation is (on average) $40.1 \%$. Quintic and septic convolution are, respectively, $42.3 \%$ and $44.8 \%$ better than linear interpolation, but only $3.7 \%$ and $7.8 \%$ better than cubic convolution.

\section{V.C Discussion of the Results}

In the subpixel translation, rotation, and magnification experiments, the actual magnitude of the mean square error for a specific image depends on the information contained in the image. If large parts of the image are highly structured, the mean square error will be large, since the largest errors are made at sharp transitions (edges). This is the case in e.g. the BAboon and Airfield images. In e.g. the Flower and Peppers images, there are large more-or-less homogeneous regions and only a relatively small part of these images contains sharp transitions, which entails that the mean square error is relatively small.

We have presented the results for one specific translation vector, rotation angle and magnification factor. In order to make sure that the observed effects were not dependent on the specific choice of these quantities, we have carried out experiments with several other vectors, angles and factors. The results of these experiments were all very consistent with the results presented here.

As can be gathered from the results of the different experiments, the precise improvement of higher-order schemes with respect to lower-order ones depends on the choice for the error measure. In general it can be stated that the specific situation in which an interpolation scheme is to be chosen also determines the error measure that must be selected in order to describe the merits of that scheme for the application. In fact, it is the type of application that determines whether a higher-order scheme is to be preferred. The experiments presented in this paper show that in all cases, the improvement of quintic or septic convolution over cubic convolution is far less significant than that of cubic convolution over linear interpolation.

Finally it should be noted that in a particular situation, the type of operations and the type of images to which they are to be applied may also determine the optimality criterion for deriving the free parameter $\alpha$. In this paper, we have chosen a criterion that has been shown to yield the most mathematical precise interpolation $[4,8]$, i.e., for which the Taylor series expansion of the interpolant will be equal to that of the original signal in as many terms as possible. In a general description of these polynomial kernels, this is the most appropriate choice.

\section{Conclusions}

In this paper, the sinc-approximating symmetrical piecewise $n$ th-order polynomial kernels were presented, which can be used for the reconstruction of $N$-dimensional images. After a derivation proving that reconstruction (or interpolation) of $N$-dimensional signals can be carried out by $N$ successive one-dimensional interpolations, and the presentation of the concept of symmetrical piecewise $n$ th-order polynomial kernels, the linear, cubic, quintic and septic convolution kernels were derived.

An objective, quantitative comparison of the performance of the four interpolation kernels was obtained by analyzing the spatial and spectral behavior of the kernels according to several measures, as well as by using them in subpixel translation, rotation and magnification experiments, applied to a number of real-life test images. The results 
of these experiments show, very consistently, that the errors made by cubic convolution are substantially smaller than those made by linear interpolation. However, higher-order schemes yield only marginal improvement, at an increased computational cost.

\section{Acknowledgments}

The research described in this paper was carried out at the Image Sciences Institute, University Medical Center Utrecht (UMCU), the Netherlands, and was financially supported by the Netherlands Ministry of Economic Affairs.

\section{References}

[1] A. Antoniou, Digital Filters: Analysis, Design, and Applications, 2nd ed., McGraw-Hill, New York, NY, 1993.

[2] T. E. Boult \& G. Wolberg, "Local Image Reconstruction and Subpixel Restoration Algorithms", CVGIP: Graphical Models and Image Processing, vol. 55, no. 1, 1993, pp. 63-77.

[3] A. J. Jerri, "The Shannon Sampling Theorem-Its Various Extensions and Applications: A Tutorial Review", Proceedings of the IEEE, vol. 65, no. 11, 1977, pp. 1565-1596.

[4] R. G. Keys, "Cubic Convolution Interpolation for Digital Image Processing", IEEE Transactions on Acoustics, Speech, and Signal Processing, vol. 29, no. 6, 1981, pp. 1153-1160.

[5] H. Kwakernaak \& R. Sivan, Modern Signals and Systems, Information and System Sciences Series, Prentice-Hall, Englewood Cliffs, NJ, 1991.

[6] H. Nyquist, "Certain Topics in Telegraph Transmission Theory", Transactions of the American Institute of Electrical Engineers, vol. 47, 1928, pp. 617-644.

[7] S. K. Park \& R. A. Schowengerdt, "Image Sampling, Reconstruction, and the Effect of Sample-Scene Phasing", Applied Optics, vol. 21, no. 17, 1982, pp. 3142-3151.

[8] S. K. Park \& R. A. Schowengerdt, "Image Reconstruction by Parametric Cubic Convolution", Computer Vision, Graphics and Image Processing, vol. 23, no. 3, 1983, pp. 258-272.

[9] J. A. Parker, R. V. Kenyon, D. E. Troxel, "Comparison of Interpolating Methods for Image Resampling", IEEE Transactions on Medical Imaging, vol. 2, no. 1, 1983, pp. 31-39.

[10] S. E. Reichenbach \& S. K. Park, "Two-Parameter Cubic Convolution for Image Reconstruction", in Visual Communications and Image Processing, vol. 1199 of Proceedings of SPIE, The International Society for Optical Engineering, Bellingham, WA, 1989, pp. 833-840.

[11] S. S. Rifman, "Digital Rectification of ERTS Multispectral Imagery", in Proceedings of the Symposium on Significant Results Obtained from the Earth Resources Technology Satellite-1, vol. 1, section B, NASA SP-327, 1973, pp. 1131-1142.

[12] C. E. Shannon, "Communication in the Presence of Noise", Proceedings of the Institution of Radio Engineers, vol. 37, no. 1, 1949, pp. 10-21.

[13] G. Timmens, "Resampling of Multidimensional Image Data", Master's thesis, Delft University of Technology, Delft, 1991.

[14] E. T. Whittaker, "On the Functions which are Represented by the Expansions of InterpolationTheory", Proceedings of the Royal Society of Edinburgh, vol. 35, 1915, pp. 181-194.

[15] G. Wolberg, Digital Image Warping, IEEE Computer Society Press, Washington, DC, 1990. 